\title{
Perioperative Evaluation and Outcomes of Major Limb Replantations with Ischemia Periods of More Than 6 Hours
}

\author{
Thrivikrama Padur Tantry, MD ${ }^{1}$ Dinesh Kadam, MS, MCh, DNB ${ }^{2}$ Sunil P. Shenoy, MS, MCh, DNB ${ }^{3}$ \\ Sanath Bhandary, MS, MCh² Karunakara K. Adappa, MD ${ }^{1}$
}

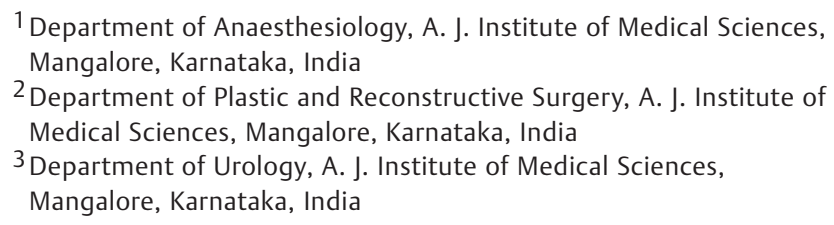

Address for correspondence and reprint requests Dinesh Kadam, MS, MCh, DNB, Department of Plastic and Reconstructive Surgery,

A. J. Institute of Medical Sciences and A. J. Hospital and Research Centre, Kuntikana Mangalore, Karnataka 575004, India

(e-mail: drkadam@yahoo.co.in).

J Reconstr Microsurg 2013;29:165-172.

\begin{abstract}
Keywords

- major replantation

- reperfusion syndrome

- prolonged ischemia

Early revascularization is cardinal for successful replantation of proximal limb amputations. Prolonged ischemia time potentially leads to reperfusion syndrome and morbidity. The dilemma persists regarding safe duration of cold ischemia time for replantation. The study was conducted to evaluate retrospectively the outcomes of major replantation in terms of limb survival, reperfusion events, morbidity, and potential mortality with respect to the ischemia time and level of amputations. Fourteen patients with proximal amputations at the arm, elbow, and forearm with total ischemia time beyond 6 hours were replanted. All had warm ischemia time of less than 2 hours and were closely monitored to record and correct reperfusion events. Nine out of 14 limbs survived. Five patients had reperfusion events. Proximal limb amputations with larger muscle mass were at higher risk of developing reperfusion syndrome and such events had higher chances of limb loss. Major limb amputations within 2 hours of warm ischemia time even with prolonged cold ischemia can be successfully replanted with closed perioperative monitoring.
\end{abstract}

Traumatic amputations of the extremities are being increasingly encountered as a fallout of industrialization, construction activity, and increasing societal violence. Advancements in reconstructive microsurgical techniques, anesthesia, and critical care have drastically altered the outlook for replantation of amputated limbs. In proximal amputations, early revascularization is cardinal to the anatomical and functional survival of replanted extremities or their parts, despite the well-documented dangers posed by reperfusion of ischemic limbs, including multisystem organ failure and death. ${ }^{1-3}$

Critical issues influencing decision-making of major replantations include level of amputation, duration of ischemia time, extent of crushing and contamination of the parts, experience of the surgical and anesthetic teams, and available infrastructure. Anxious relatives and compelling socioeconomic factors also influence decision-making in unfavorable situations.

The principal determining factor, however, remains the warm ischemia time. Replantation is not considered in amputations beyond 2 to 3 hours of the injury due to irreversible neuromuscular changes. Yet, amputated limbs with muscle mass have been successfully replanted up to 12 hours after injury, provided the limbs are cold-preserved. ${ }^{4}$ However, in a situation where warm ischemia time remains 2 hours or less and the limb is cold preserved, definitive guidelines regarding decisions to replant are not available. Dilemma still remains received

July 1,2012

accepted after revision

September 7, 2012

published online

January 25, 2013
Copyright $\odot 2013$ by Thieme Medical Publishers, Inc., 333 Seventh Avenue, New York, NY 10001, USA. Tel: +1(212) 584-4662.
DOI http://dx.doi.org/ 10.1055/s-0032-1331143. ISSN 0743-684X. 
regarding the safe limit for cold ischemia time in relation to the level of amputation and possible reperfusion injury, morbidity, limb survival, functional outcome, and potential mortality following such procedures.

This retrospective noncomparative interventional study projects our results on replantation of single limb segments in 14 patients between 2006 and 2010, with total ischemia periods more than 6 hours. Our results indicate that replantation of severed extremities can be performed with acceptable results in situations where the warm ischemia time is less than 2 hours but the cold ischemia time is prolonged, provided strict standards of perioperative care are ensured. The decision for replantation should be individualized. The value of intensive monitoring and critical care by the clinician is stressed.

\section{Patients and Methods}

Fourteen patients who underwent major limb replantations between 2006 and 2010 after having the severed segments subjected to ischemia times more than 6 hours were included in this study. All patients were operated on by a single surgeon and his team, and anesthetic and supportive perioperative care was provided by a single anesthesiologist and his team. Photographic documentation was done in all cases.

In the emergency room, patients were stabilized hemodynamically and administered broad-spectrum antibiotics and analgesics. Four patients on presentation had hypotension due to traumatic blood loss, requiring colloids. All patients had radiological examination (stump and the amputated part), total white cell count, coagulation profile, serum electrolytes, renal parameters, and electrocardiogram done. All had a central venous line and urethral catheter inserted. The time, type, extent of injury of the limb, contamination, crush of devitalized parts, duration of ischemia, and the mode of preservation of the amputated part during the transport were tabulated. The total ischemia period considered was the time interval from the time of injury to the time of establishment of arterial and venous anastomoses. This was further divided into warm and cold ischemia periods based on the limb preservation in hypothermic environment. Cold ischemia time included hypothermic preservation during transport, preoperative and intraoperative cooling of the limb.

All amputated parts were perfused preoperatively with cold heparinized ringer lactate solution, cooled externally with ice cold saline pads, and kept in a cold environment prior to and during the procedure. Anesthesia was induced with thiopentone $(n=9)$, propofol $(n=4)$ or ketamine $(n=1)$ and maintained using halothane $(n=2)$, isoflurane $(n=11)$, sevoflurane $(n=1)$ and nitrous oxide-oxygen gas mixture along with an opioid. Intraoperative monitoring included arterial pressure $(n=9)$ and central venous pressure (CVP, $n=14$ ), urine output, end tidal $\mathrm{CO}_{2}$ (ETCO2), electrocardiography, oxygen saturation ( $\mathrm{SpO} 2$ ), temperature, blood gas analysis, serum electrolytes, and hemoglobin estimation. Surgical techniques employed during replantation included debridement, bone shortening, skeletal fixation with plating or intramedullary nail/pins, soft tissue/tendon repair of flexor and extensor group followed by vascular anastomosis and nerve repair. A similar sequence of vascular anastomosis was followed in all patients, with initial repair of a single vein (V1) and a single artery (A1). Vein grafts were used in four patients. A second vein (V2) for repair was identified. At this point the arterial clamp (A1) was opened and venous bleed (V2) was allowed for 5 minutes. Second venous (V2) anastomosis was done following release of V1 clamp. Subsequently, a second artery (A2) or third vein (V3) anastomosis was performed.

During the flush out of venous channels, CVP was monitored and maintained with colloids and crystalloids. Prophylactic bicarbonates ( 1 to $2 \mathrm{meq} / \mathrm{kg}$ ) were injected intravenously at this time. Arterial blood gases, hemoglobin, coagulation profile (prothrombin time [PT], international normalized ratio [INR], activated partial thromboplastin time [aPTT], platelets), and serum electrolytes were analyzed within 30 minutes of the completion of anastomosis. Repeat blood gas analyses and coagulation profiles were done when indicated. The surgical procedure was concluded with hemostasis, fasciotomy, and skin approximation. Intraoperative urine output was noted on an hourly basis. A dose of heparin (50 to $100 \mathrm{IU} / \mathrm{kg}-1$ ) was administered in patients when the bleeding was not excessive. Excessive bleeding was purely a subjective observation by the clinician. Intraoperative usage of crystalloids and colloids (dextran-40, blood, and blood products) was noted.

Postoperatively, patients were monitored for blood gases, hemoglobin, white cell counts, coagulation profiles, and electrolytes at regular intervals and abnormalities were corrected. Re-explorations for vascular cause and a second surgical procedure for wound cover with free flap or skin graft were performed when indicated. The limb was considered to have survived whenever the patient was discharged from the hospital with a viable replanted limb segment (-Fig. 1 and 2).

\section{Statistical Analysis}

Statistical analysis was performed using MedCalc software (version 9.3.6.0, Belgium). Different ischemia durations with respect to viability of limb and the level of amputation and to the reperfusion events were analyzed using Mann-Whitney U test. Evaluation of association of variables (contamination, level of amputation, viability) with respect to limb survival and reperfusion events was done using Fisher's exact test. Role of crush factor was analyzed with chi-square test (with limb survival and reperfusion events). A value of $p<0.05$ was considered statistically significant.

\section{Results}

Fourteen patients, including 5 females of various ages, had injury to the upper/lower limb with complete amputation (-Table 1). Durations of warm, cold, and total ischemia and durations of intensive care unit (ICU) and hospital stays are detailed in - Table 2.

All intraoperative blood gas readings showed normal $\mathrm{pH}$ except in one patient who showed acidosis. All patients required blood and blood component transfusions, and six 


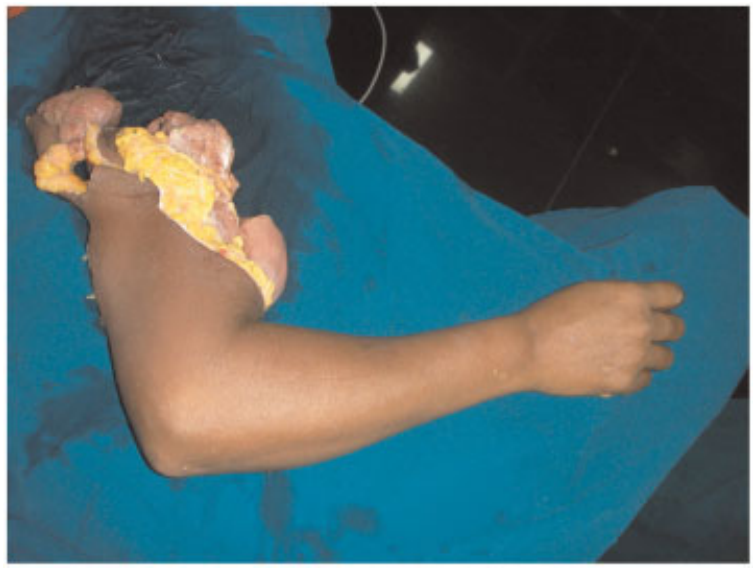

Fig. 1 Traumatic crush amputation at arm level in a 35-year-old woman (Patient 14) due to road traffic accident. Warm and cold ischemia time were 120 minutes and 660 minutes, respectively, and there was no reperfusion event.

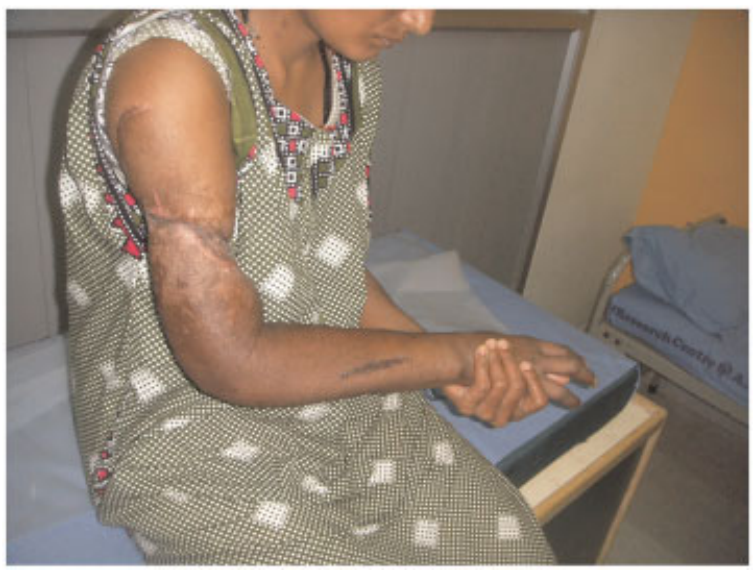

Fig. 2 Four months postop following replantation.

patients required additional colloid (hetastarch 6\%) transfusion. Heparin was used in 11 patients, and low molecular weight heparin in 1 . None had altered PT, INR, or aPTT.

Duration and type of ischemia were comparable in groups of patients with respect to limb survival ( $p>0.05$, MannWhitney U test, - Table 3). A statistically significant higher warm ischemia was observed in patients who developed reperfusion events $(p=0.038)$. Patients with reperfusion events had higher limb loss $(p=0.023$, Fisher's exact test, odds ratio 32, - Table 4 ). The more proximal the amputation, the more were such events observed ( $p=0.023$, odds ratio 0.031, - Table 3). However, contamination and crush had no significant association with adverse events or limb survival ( $p>0.05$, Chi-square test, - Table 2 and 3). Higher perioperative transfusion requirement was observed with higher level of amputation ( $p=0.039$, Mann-Whitney U test, - Table 5).

\section{Specific Patient Related Intraoperative Events}

Event 1 Hypotension and atrial fibrillation with acidosis was observed in a 38-year-old male with upper arm amputation. This event appeared 30 minutes following the vascular anas- tomosis and persisted for 20 minutes. Arterial pH was 7.29 at the 30th minute, which normalized in the 60th minute sample. Electrolyte readings were normal in this patient. Bicarbonate infusions of $200 \mathrm{meq}$ were used to correct acidosis. Dopamine and adrenaline were required for maintenance of blood pressures subsequently.

Event 2 An episode of acute bronchospasm with increased ETCO2 and high airway pressures was recorded immediately following establishment of vascularity of an arm replantation in a 3-year-old female. There was no associated hypotension and the child responded to salbutamol nebulization and intravenous deriphyllin.

Event 3 Unexplained hypotension episodes requiring pharmacological intervention beyond volume replacement were recorded in two patients (36- and 20-year-old male patients with upper forearm and upper arm amputation, respectively). CVPs were adequate in these patients. Dopamine alone or in combination with adrenaline was used and continued postoperatively for a day.

Event 4 Acute metabolic acidosis followed by hypotension (79/56 $\mathrm{mm} \mathrm{Hg}$ ) was observed in a 35-year-old female. A blood gas picture of $\mathrm{pH} 6.961, \mathrm{pCO}_{2} 27.2, \mathrm{pO}_{2} 109.1 \mathrm{~mm} \mathrm{Hg}$, and $\mathrm{HCO}_{3}$ of 6.0 was observed at the fourth hour of completion of replantation. Rapid correction of acidosis with bicarbonate (200 meq), treatment of hypotension with volume replacement and inotropes (dopamine), and elective mechanical ventilation were done. Repeat blood gas analysis showed $\mathrm{pH}$ of 7.348 and $\mathrm{HCO}_{3}$ of 15.2 after 1.5 hours, which was successfully corrected. She was extubated after 24 hours and remained stable with satisfactory limb vascularity. However, she developed transfusion-related acute hemolytic reaction following packed-cell transfusion on the sixth postoperative day, requiring vasopressors and mechanical ventilatory support. Serum potassium levels were normal before the event. This patient died and medicolegal autopsy did not reveal any causative finding. The mortality was concluded as a blood transfusion-related event.

\section{Postoperative Period}

Three patients required mechanical ventilation due to delayed recovery from anesthesia ( $<4$ hours), acute metabolic acidosis, and hemodynamic instability (24 hours). Blood and component therapy were required in all patients. All patients had adequate urine output $>1 \mathrm{ml} / \mathrm{kg} /$ hour during the first 24 hours after replantation. Myoglobinuria was observed in one, but none showed elevated creatinine levels.

Surgical re-exploration was required in 3 patients for anastomotic blowout (2) and vascular thrombosis (1). Of the 13 surviving patients, 4 had limb loss within the 4week period due to thrombosis of vessels (3) and local sepsis (1).

\section{Discussion}

Successful replantation of amputated limb segments primarily depends on the level of amputation and early revascularization. Prolonged ischemia time particularly in proximal amputations is considered detrimental to replantation due 
Table 1 Patients with details of level of amputation, warm and cold ischemia time, complications, and final outcome

\begin{tabular}{|c|c|c|c|c|c|c|}
\hline $\begin{array}{l}\text { Patient } \\
\text { no. }\end{array}$ & $\begin{array}{l}\text { Age } \\
\text { (years)/ } \\
\text { gender }\end{array}$ & $\begin{array}{l}\text { Level of } \\
\text { amputation }\end{array}$ & $\begin{array}{l}\text { Warm } \\
\text { ischemia } \\
\text { time (min) }\end{array}$ & $\begin{array}{l}\text { Cold ischemia } \\
\text { time (min) }\end{array}$ & $\begin{array}{l}\text { Reperfusion } \\
\text { events/complications }\end{array}$ & Final outcome \\
\hline 1 & $36 / \mathrm{M}$ & Proximal forearm & 105 & 390 & Hypotension & Limb loss \\
\hline 2 & $2 / \mathrm{M}$ & Proximal Forearm & 20 & 420 & & Limb Survived \\
\hline 3 & $15 / M$ & Wrist & 55 & 115 & & Limb Survived \\
\hline 4 & $30 / \mathrm{M}$ & Distal forearm & 30 & 435 & & Limb Survived \\
\hline 5 & $12 / F$ & Proximal forearm & 30 & 550 & & Limb Survived \\
\hline 6 & $20 / \mathrm{M}$ & Proximal arm & 90 & 900 & Hypotension/re-exploration & Limb loss \\
\hline 7 & $3 / F$ & Mid arm & 120 & 360 & Bronchospasm/re-exploration & Limb loss \\
\hline 8 & $38 / \mathrm{M}$ & Distal arm & 80 & 430 & $\begin{array}{l}\text { Atrial fibrillation, hypotension/ } \\
\text { re-exploration }\end{array}$ & Limb Survived \\
\hline 9 & $20 / M$ & Proximal leg & 20 & 435 & & Limb loss \\
\hline 10 & $52 / \mathrm{M}$ & Wrist & 60 & 920 & & Limb Survived \\
\hline 11 & $36 / F$ & Mid forearm & 100 & 325 & & Limb Survived \\
\hline 12 & $37 / \mathrm{M}$ & Proximal arm & 100 & 785 & Hypotension & Limb Survived \\
\hline 13 & $35 / F$ & Mid arm & 145 & 435 & $\begin{array}{l}\text { Acute met acidosis in } \\
\text { immediate post op/ } \\
\text { re-exploration }\end{array}$ & Mortality \\
\hline 14 & $35 / F$ & Proximal arm & 110 & 670 & & Limb Survived \\
\hline
\end{tabular}

Table 2 Patients data

\begin{tabular}{|c|c|}
\hline \multicolumn{2}{|l|}{ Demographic data: } \\
\hline Total number of patients & 14 \\
\hline Age (years, mean \pm sd) & $26.5 \pm 14.7$ \\
\hline Weight $(\mathrm{kg}$, mean $\pm \mathrm{sd})$ & $50 \pm 20.18$ \\
\hline Sex ratio (male:female) & 9:05 \\
\hline \multicolumn{2}{|l|}{ Level of amputation: } \\
\hline Wrist & 2 \\
\hline Forearm & 4 \\
\hline Elbow & 2 \\
\hline Arm & 5 \\
\hline Leg & 1 \\
\hline \multicolumn{2}{|c|}{ Ischemia times (minutes, mean $\pm \mathrm{SD}$, median): } \\
\hline Warm ischemia & $76.07 \pm 40.44,85$ \\
\hline Cold ischemia & $512.86 \pm 228.71,435$ \\
\hline Total ischemia & $602.5 \pm 213.53,502.5$ \\
\hline \multicolumn{2}{|l|}{ Other perioperative details: } \\
\hline ICU days (mean $\pm \mathrm{sd}$ ) & $13.86 \pm 9.65$ \\
\hline IP days (mean \pm sd) & $41.86 \pm 27.09$ \\
\hline $\begin{array}{l}\text { Patients discharged with } \\
\text { viable limb }\end{array}$ & 9 \\
\hline Mortality & 1 \\
\hline
\end{tabular}

to irreversible tissue changes, possibility of reperfusion injury events, and potential morbidity and mortality. ${ }^{1}$ There is a paucity of literature on replantation studies relating to prolonged ischemia time, but successful revascularization of amputated upper limbs has been reported after 10 hours of warm ischemia. ${ }^{5}$

Patients or their family members plead for replantation, irrespective of the prognosis or danger to life. Neuronal and muscle tissues tolerate ischemia poorly and functional recovery of a viable limb therefore may be compromised when ischemia time gets prolonged. ${ }^{6}$ The optimal maximum cold ischemia time for proximal replantations is not yet clearly defined. Since our study included only patients with prolonged ischemia time (beyond 6 hours), we studied the factors influencing this crucial parameter, with specific reference to our conditions. All our patients had warm ischemia time less than 2 hours, which is well within accepted norms. This was indeed an encouraging signal indicating increasing awareness of people and health care personnel even in remote areas to the need for and basic knowledge of coldpreserving amputated limbs for replantation. In a few cases, telephone guidance was given to the patient's family or health care personnel on the preservation technique. The greater

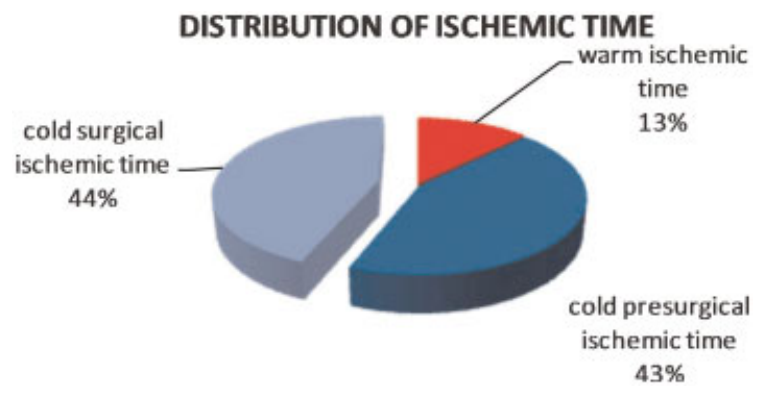

Fig. 3 Distribution of ischemia time. 


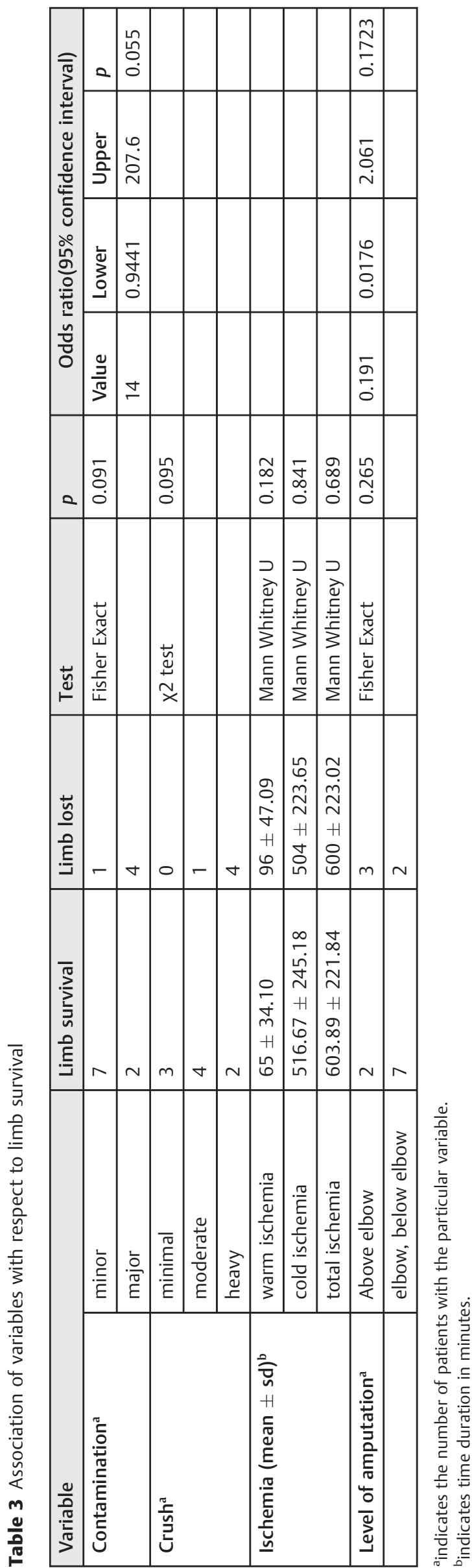

problem faced by most patients was the longer presurgical cold ischemia time (56\% of total time till re-establishment of vascularity, - Fig. 3). Factors affecting overall presurgical ischemia times included deficiency of quick transportation facilities causing delay in reaching the primary or tertiary hospital and time for primary stabilization of patient by the referring hospital. Understandably, rural areas lack facilities and expertise for microsurgical revascularization, but in urban areas too, only select tertiary care centers deal with such procedures, and considerable time is lost due to interreferral time delay between them. Evaluation of injuries, stabilizing the patient hemodynamically, making a decision to replant, and apprising relatives on the cost and outcomes of the procedure involves some unavoidable delay.

Prolonged ischemia time is associated with greater changes in cellular metabolism, especially in muscles, and can produce permanent damage and reperfusion syndrome. ${ }^{1,7}$ Waikakul and collegues ${ }^{8}$ in one of the largest reported limb replantation series (186 patients) described postoperative reperfusion syndrome in $5.4 \%$ of patients. The authors, however, have neither mentioned adverse intraoperative events in this selected group nor come to a conclusion on the clear linear correlation between prolonged ischemia time and the frequency and severity of the syndrome. All our patients had total limb ischemia time more than 6 hours. The patients who developed intraoperative/ early postoperative events had $\geq 8$ hours of total ischemia. Reperfusion events observed in our patients were hypotension, bronchospasm, acidosis, and atrial fibrillation.

Patients with hypotension who required inotropic support had adequate $\operatorname{CVP}(\geq 10)$, indicating normovolumic hypotension. Hypotension occurring following revascularization could be related to (1) redistribution of blood flow; (2) systemic effects of chemical mediators, toxic products, and acidosis; or (3) excessive bleeding following coagulation failure and disseminated intravascular coagulation (DIC). ${ }^{2}$ Cytokinins produced in the organ beds of anoxic limbs may be a major factor for hypotension. However, sepsis and crush syndrome could also contribute to this effect. Low blood pressure could potentially lead to circulatory stagnation and thrombosis of freshly anastomosed vessels. Hence maintaining adequate blood pressure is absolutely essential in perioperative period. If volume resuscitation proves inadequate, bicarbonate, a vasopressor, vasoconstrictor, or an inotrope should be used to optimize blood pressure. ${ }^{9}$

Intraoperative atrial fibrillation was attributable to reperfusion. Metabolic acidosis on establishment of vascular continuity may trigger arrhythmias, as observed in our patient. Combined hyperchloremic lactic acidosis may be a possibility; we have not measured blood lactate levels. Prophylactic bicarbonate supplements were administered intraoperatively in anticipation of acidosis in most of our patients.

Rapid development of acidosis and hyperkalemia following replantation is reported, ${ }^{10}$ although we observed no such hyperkalemic response. Acute intraoperative metabolic acidosis or other adverse events occasionally require perioperative re-amputation as a life-saving measure. ${ }^{8,10}$ Acute early postoperative metabolic acidosis with hemodynamic 


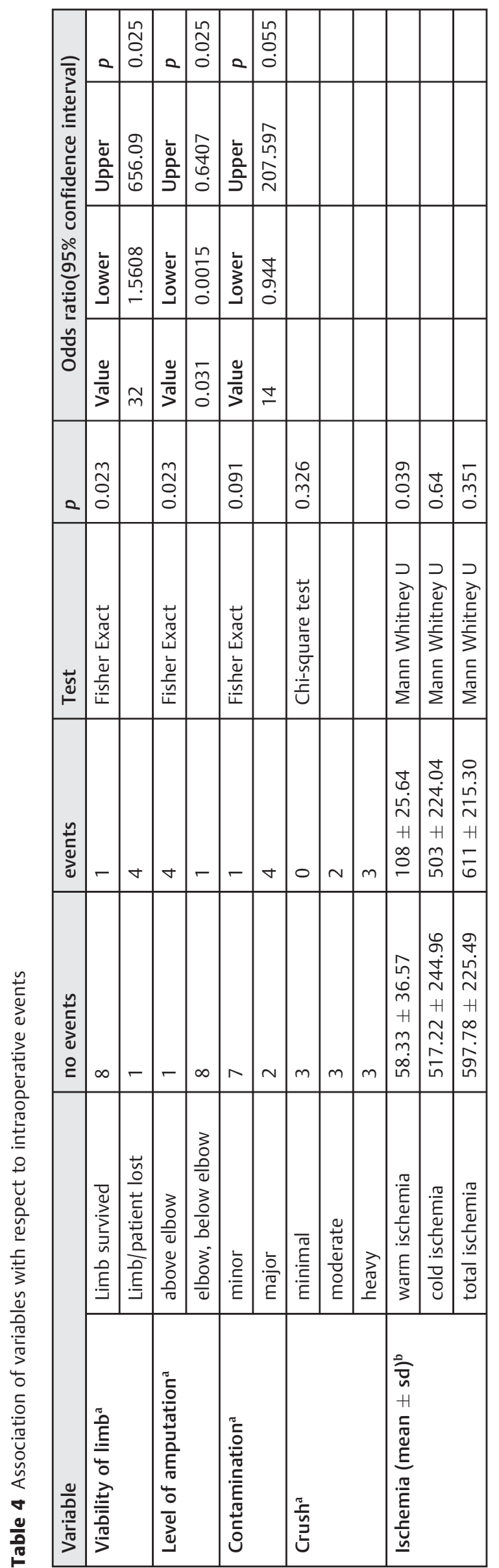

instability probably induced by reperfusion needed aggressive correction to stabilize the patients. None of our patients needed re-amputation despite relatively prolonged ischemia time.

We found no specific mention in the literature with regards to bronchospasm as a manifestation of reperfusion syndrome. This event, observed in a child in the absence of a previous history of asthma and occurring within 10 minutes of revascularization, cannot be anything but a reperfusionrelated event. Complement $(\mathrm{Ca})$ is the key mediator of reperfusion injury, and its activation results in release of chemotactic agent (C5a) and anaphylatoxins (C3a and $\mathrm{C5a}$ ) that further induce degranulation of mast cells with release of histamine and other mediators that induce bronchospasm. ${ }^{11-13}$

Two specific adverse outcomes, reperfusion events and limb loss, were studied to correlate with contributing factors. Both warm ischemia and the level of amputation showed a strong association with these adverse events. Four out of five patients with reperfusion events had proximal amputations, and patients with reperfusion events had higher mean warm ischemia time. A statistically significant association between limb loss and reperfusion syndrome was observed in proximal amputations with prolonged warm ischemia time. Interestingly, prolonged cold ischemia was not significantly associated with either reperfusion events or limb loss. A higher mean warm ischemia was seen in limb loss patients. The warm ischemia time, which is within acceptable limits of 2 hours alone, is unlikely to produce adverse outcome unless followed by prolonged cold ischemia time. The total cold ischemia time, comprising cold-preserved transport time and external cooling during the surgical procedure, might not have maintained the uniform temperature of $4^{\circ} \mathrm{C}$, resulting in a significant unspecified warm ischemia time of the limb. The level of amputation by itself had no bearing on limb survival. Similarly, severity of crush and contamination had no relation to either reperfusion injury or limb survival, which could be attributed to adequate wound debridement prior to the replantation. It is therefore only logical to conclude that more chemical mediators are liberated into the systemic circulation from an amputated limb segment of larger diameter with longer ischemia time in the aftermath of revascularization, resulting in a higher chance of reperfusion injury. Furthermore, reperfusion-induced local tissue injury has a deleterious effect on the survival of the limb even though the systemic manifestations of such injury are managed effectively. The resulting endothelial injury and hypotension has a higher chance of anastomotic thrombosis, which was seen in all four patients who had reperfusion events requiring surgical re-exploration in an attempt to salvage the limbs.

Perioperative transfusion requirements had significant association with the level of amputation. Higher blood (and products) transfusion requirements solely did not represent intraoperative loss; repeated transfusion requirements were observed at a later date (subsequent surgery). Several authors have described the sequential technique of venous flow clamping and release from the partially replanted limb for a period of 10 to 20 minutes to reduce the systemic ingress of 
Table 5 Association of variables with respect to the level of amputation

\begin{tabular}{|c|c|c|c|c|}
\hline Variable (mean $\pm \mathrm{sd}$ ) & above elbow & elbow, below elbow & Test & $p$ \\
\hline Warm ischemia ${ }^{a}$ & $109 \pm 25.59$ & $57.78 \pm 35.72$ & Mann Whitney U & 0.028 \\
\hline Transport time ${ }^{\mathrm{a}}$ & $402 \pm 238.45$ & $301.67 \pm 230.39$ & Mann Whitney U & 0.286 \\
\hline Cold presurgical ischemia time & $293 \pm 246.64$ & $243.89 \pm 217.86$ & Mann Whitney U & 0.505 \\
\hline Cold surgical ischemia time ${ }^{\mathrm{a}}$ & $266 \pm 78.69$ & $264.44 \pm 57.47$ & Mann Whitney U & 0.842 \\
\hline Total cold ischemia time ${ }^{a}$ & $559 \pm 223.73$ & $508.33 \pm 209.55$ & Mann Whitney U & 0.64 \\
\hline Average transfusions ${ }^{b}$ & $19.4 \pm 9.96$ & $7.33 \pm 5.66$ & Mann Whitney U & 0.039 \\
\hline
\end{tabular}

andicates time duration in minutes.

bindicates number of blood or blood product units.

toxins from the damaged ischemic tissues. ${ }^{14-16}$ Arterial flow to the limb was maintained during this period and venous drainage was facilitated by allowing bleeding from unanastomosed veins. We allowed an average of 5 minutes of venous bleed egress from the unanastomosed vein. The anastomosed vein, which was kept clamped initially, was declamped after 5 minutes, thereby preventing excessive blood loss. The fine balance between excessive bleeding causing hypotension and sudden systemic entry of venous blood leading to adverse reperfusion events is thus maintained. One of the reported studies totally avoided venous blood drainage during surgery owing to the enormous technical effort needed and the deleterious effects of volume depletion associated with it. ${ }^{14}$

Although the risk of an individual patient succumbing to reperfusion syndrome is very real and should on no account be ignored, we observe that replantation of amputated limbs with warm ischemia time within 2 hours even with prolonged cold ischemia time can be successfully performed. The highest degree of anesthetic and surgical efforts and care is required to check morbidity and risks associated with the procedure. Though we used aggressive statistical analysis, the incidence and outcome would not vary. The end results of significant limb survival indeed were gratifying. The functional outcome of the surviving reattached limbs in our study is under evaluation.

\section{References}

1 Beyersdorf F. Protection of the ischemic skeletal muscle. Thorac Cardiovasc Surg 1991;39:19-28

2 Eltzschig HK, Collard CD. Vascular ischaemia and reperfusion injury. Br Med Bull 2004;70:71-86

3 Blaisdell FW. The pathophysiology of skeletal muscle ischemia and the reperfusion syndrome: a review. Cardiovasc Surg 2002;10: $620-630$
4 Buncke GM. Replantation and revascularization. Part 1, volume VII. In: Mathes SJ, ed. Plastic Surgery. 2nd ed. Philadelphia: Saunders Elsevier; 2006:565-585

5 Merican AM, Kwan MK, Cheok CY, Wong EL, Sara TA. Successful revascularisation of near total amputation of the upper limb after ten hours of warm ischaemia. Med J Malaysia 2005;60:218-221

6 Burkhardt GE, Gifford SM, Propper B, et al. The impact of ischemic intervals on neuromuscular recovery in a porcine (Sus scrofa) survival model of extremity vascular injury. J Vasc Surg 2011;53: 165-173

7 Carden DL, Korthuis RJ. Mechanisms of postischemic vascular dysfunction in skeletal muscle: implications for therapeutic intervention. Microcirc Endothelium Lymphatics 1989;5:277-298

8 Waikakul S, Vanadurongwan V, Unnanuntana A. Prognostic factors for major limb re-implantation at both immediate and long-term follow-up. J Bone Joint Surg Br 1998;80:1024-1030

9 Murray MJ, Shine TSJ. Surgery of thoracic and abdominal aorta. In: Kaplan JA, ed. Vascular Anaesthesia. 2nd ed. Philadelphia: Elsevier Churchill Livingstone; 2004:212-213

10 McCutcheon C, Hennessy B. Systemic reperfusion injury during arm replantation requiring intraoperative amputation. Anaesth Intensive Care 2002;30:71-73

11 Smith GW, McArthur CJ, Simpson IJ. Circulating immune complexes in myocardial infarction. J Clin Lab Immunol 1983;12: 197-199

12 Khalil AA, Aziz FA, Hall JC. Reperfusion injury. Plast Reconstr Surg 2006;117:1024-1033

13 Lazarus B, Messina A, Barker JE, et al. The role of mast cells in ischaemia-reperfusion injury in murine skeletal muscle. J Pathol 2000;191:443-448

14 Wilhelm MP, Schlensak C, Hoh A, et al. Controlled reperfusion using a simplified perfusion system preserves function after acute and persistent limb ischemia: a preliminary study. J Vasc Surg 2005;42:690-694

15 D'Addato M, Pedrini L, Bertoni M, et al. Traumatic amputation of the upper limb: replantation of the arm. J Trauma 1989;29: 876-879

16 Beyersdorf F, Mitrev Z, Ihnken K, et al. Controlled limb reperfusion in patients having cardiac operations. J Thorac Cardiovasc Surg 1996;111:873-881 